\title{
Article
}

\section{Stabilization of switched linear systems with control constraints based on EA}

\author{
Houssem HOSNI ${ }^{1, *}$, Walid BEN MABROUK ${ }^{1}$ and Noureddine LIOUANE 1 \\ 1 Laboratory of Automatic, Signal Processing and Image (LARATSI), National Engineering School of \\ Monastir (ENIM), Rue Ibn El Jazzar, Monastir 5019, Tunisia; walid.mabrouk@enim.rnu.tn (W.M); \\ noureddine.liouane@enim.rnu.tn (N.L.) \\ * Correspondence: hosny.houssem@gmail.com (H.H.)
}

\begin{abstract}
In this paper, we address the problem of stabilization of switched linear systems. The idea is to look for a state feedback control law using evolutionary algorithms (EA) in order to assure the stability of the switched linear systems under control constraints. In some cases when states are not available and only outputs are measurable, the previous method is applied to design an output feedback controller which stabilizes the system. Both stabilizing controllers are developed using deferential evolution and genetic algorithm. Two numerical examples illustrate our proposed theory and point out the effectiveness of our proposed approaches.
\end{abstract}

Keywords: Stabilization, control constraints, evolutionary algorithms, switched linear system.

\section{Introduction}

Switched system is a family of hybrid dynamical systems that consists of a finite number of subsystems and a logical rule that assures switching between these subsystems. Switched systems have numerous applications in the field of control of mechanical systems, process control, power systems, automotive industry, aircraft and traffic control, and many other domains. [1][2][3].

The control of switched systems is an important topic in research. Up to now, most of existing literatures related to stability of switched systems focused on Lyapunov asymptotic stability [4-9], switched quadratic Lyapunov functions and common quadratic Lyapunov functions (CQLF) [10]. Given the importance of the existence of CQLF, many researchers and scientists worked on the conditions of its existence and non-existence [10][11]. The design of a method for finding a CQLF given its existence by solving the linear matrix inequalities (LMI). The linear matrix inequalities (LMI) solve several problems of stability and optimal control. Indeed, the multiple Lyapunov inequalities and algebraic Riccati inequality can be represented by an LMI formulation [12].

Moreover, the design of stabilizing control laws for switched linear systems was the subject of many researches. Several approaches have been considered for this problem include [13][14] for approaches using dynamic programming [15][16] to variational approaches, or [17][18] for approaches based on Lyapunov function. The problem is not easy, even numerically [19] and the design of a stabilizing law based on the optimization of a criterion is a difficult task.

In addition, other methods based on evolutionary algorithms were used to search a stabilizing control law for switched systems. We may mention for example the use of genetic algorithm to study the stability and stabilization of discrete switching systems [20]. Moreover, a method for calculating a common quadratic Lyapunov function CQLF based on particle swarm optimization (PSO) has 
been recently developed [21]. Furthermore, the PSO algorithm was used also for determining the non-existence of a common quadratic Lyapunov function for switched linear systems [22]. Besides, other methods for finding a CQLF was developed with the aim of improving the limitations of LMI tools, and the most outstanding is the gradient-based method [23].

Differential Evolution (DE) and Genetic Algorithm (GA) are simple stochastic optimization algorithms and evolve a population of potential solutions (individuals) in order to increase the convergence to optimal solutions. DE and GA are global search algorithms employ heuristics to allow escaping from local minima. They are optimization algorithms based on a technique derived from the genetic, and a nature evolution mechanism: crossovers, mutations, selections, they belong to the class of evolutionary algorithms.

In [24], we have studied the stability of linear switching systems without any constraint on the control. Thus, we have created a new method of finding a stabilizing control law for this class of system without any constraint. Therefore, we intend this paper to look for a stabilizing control law by state feedback and output feedback for switched linear systems under control constraints based on evolutionary algorithms.

The paper is organized as follows: Some preliminaries and problem situation are presented in section 2. In section 3, differential evolution and genetic algorithm are described. In section 4, stabilization of switched linear systems under control constraints is developed. Section 5 is devoted to algorithms implementation. Section 6 gives simulation results and comparative study on switched linear stabilization using two evolutionary algorithms shows the effectiveness of both differential evolution and genetic algorithm. Finally, section 7 provides some conclusions that end the paper.

\section{Preliminaries and problem situation}

Switched systems consist of a family of subsystems described by differential or difference equations and a switching law that manipulates switching between these subsystems.

Consider the switched linear system described by:

$$
\left\{\begin{array}{c}
\dot{x}(t)=A_{\sigma} x(t)+B_{\sigma} u(t) \\
y(t)=C_{\sigma(t)} x(t)
\end{array}\right.
$$

Where $\mathrm{A}_{\sigma(\mathrm{t})} \in \mathrm{A}=\left\{\mathrm{A}_{1}, \mathrm{~A}_{2}, \ldots, \mathrm{A}_{\mathrm{N}}\right\}, x \in \mathbb{R}^{n}$ is the state vector, $u \in \mathbb{R}^{m}$ is the control, $y \in \mathbb{R}^{p}$ is the output, $A, B$ and $C$ are constant matrices with appropriate dimensions and $\sigma(t)$ is an arbitrary signal that define commutation among elements in $A$.

We are interested by the research of the optimal state feedback and the optimal output feedback controllers which ensure the stability of the closed-loop system under control constraints using differential evolution and genetic algorithm.

\subsection{Problem 1}


Design of a state feedback control $u(t)=-K_{\sigma} x(t)$ with $K_{\sigma} \in \mathbb{R}^{m \times n}$ under control constraints using differential evolution and genetic algorithm such that the switched linear system (2.1) is stable in closed-loop.

\subsection{Problem 2}

Search the observer gain $L$ using differential evolution and genetic algorithm in order to get the control low of the static observer $u(t)=-L_{\sigma} y(t)$ with $L_{\sigma} \in \mathbb{R}$ such that closed-loop system is stable under control constraints.

\section{Differential Evolution and Genetic Algorithm}

\subsection{Differential Evolution}

The differential evolution algorithm (DE) is a stochastic optimization technique based on population of solutions, introduced by Storn and Price in 1997, in order to optimize the functions with real parameters [25-27]. This technique is characterized by its simplicity and its power simultaneously. The differential evolution algorithm is part of the evolutionary algorithms. It is inspired by genetic algorithms (crossover operator and mutation) and geometric strategies of research.

There are four processes in DE which are:

\subsubsection{Initialization}

All parameter vectors in a population are randomly initialized and evaluated using the fitness function. The initial NP D-dimensional vectors are chosen randomly and should cover the entire parameter space $x_{i}^{0}$; where $i=1,2, . ., \mathrm{NP}$. NP is size of population vectors, $\mathrm{D}$ is the number of decision variables.

\subsubsection{Mutation}

DE generates new vectors by adding the difference between two vectors to a third one. The mutant vector is generated according to:

$$
v_{i}^{t}=x_{b e s t}^{t}+F\left(x_{r 1}^{t}-x_{r 2}^{t}\right)
$$

Where $v_{i}^{t}$ is a mutant vector; $r 1, r 2$ represent random integer mutually different from index $i$; $F$ is named the scale factor, it is a real and constant factor which controls the amplification of the Best vector (practically; $0 \leq F \leq 2$ ). Figure 1 illustrate the principle of mutation. 


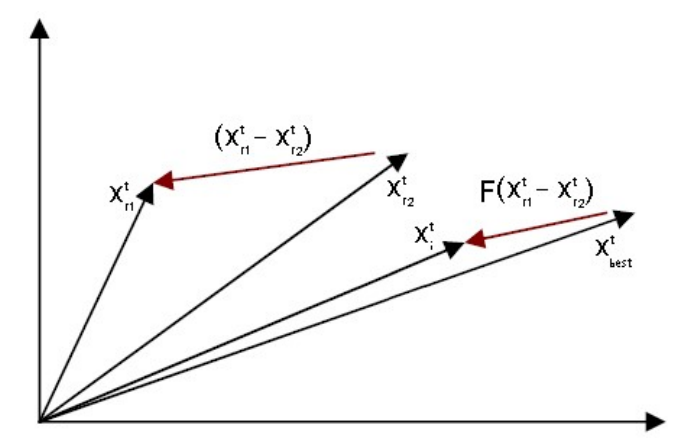

Figure 1. The principle of mutation

\subsubsection{Crossover}

Considering the each best vector $x_{b e s t}^{t}$ in the current population, a trial vector $u_{i}^{t}$ is generated by crossing the best vector with the corresponding mutant vector $v_{i}^{t}$ under a pre-specified crossover rate $C R \in\left[\begin{array}{ll}0 & 1\end{array}\right]$. The mutated vector's elements are then mixed with the elements of the predetermined best vector to yield the so-called trial vector. Moreover, choosing a set of crossover points, like crossover operator in genetic algorithms or evolution strategies, the crossover is introduced to increase the diversity of the perturbed parameter vectors. The trial vector is formulated by:

$$
u_{i}^{t}=\left\{\begin{array}{lr}
v_{i}^{t} & \text { if }(\operatorname{rand}[0,1]<C r) \\
x_{\text {best }}^{t} & \text { otherwise }
\end{array}\right.
$$

where $\operatorname{rand}[0,1]$ is an uniform random number generator with outcome $[0,1], C R$ is the crossover constant.

\subsubsection{Selection}

If the trial vector $u_{i}^{t}$ has equal or better fitness function value than that of its corresponding best vector $x_{b e s t}^{t}$, it replaces the best vector. Otherwise, the best vector remains in the next iteration. The differential evolution algorithm can be summarized in table 1.

Table 1. Differential evolution algorithm

1: Initialize of parameters of DE: Scale factor F, number of population, crossover probability $\mathrm{CR}$, number of maximum iteration $\mathrm{N}$

2: While (stop condition is not verified) do:

3: $\quad$ Select randomly $X_{r 1}^{k}, X_{r 2}^{k}$ and $X_{\text {best }}^{k}$ 
4: $\quad$ Mutation: Generate the mutant vector $X_{\text {new }}^{k}$ such as

$$
X_{\text {new }}^{k}=X_{\text {best }}^{k}+F\left(X_{r 1}^{k}-X_{r 2}^{k}\right)
$$

5: $\quad$ Crossover: Apply the crossover operator and compute the trial vector: $U_{i}^{k}$

6:

$$
U_{i}^{k}=\left\{\begin{array}{lr}
X_{\text {new }}^{k} & \text { if }(\operatorname{rand}[0,1]<C r) \\
X_{\text {best }}^{k} & \text { otherwise }
\end{array}\right.
$$

7: Selection: Evaluate trial vector for each vector

$$
X_{i}^{k+1}=\left\{\begin{array}{lr}
U_{i}^{k} & \text { if } f\left(U_{i}^{k}\right) \leq f\left(X_{i}^{k}\right) \\
X_{i}^{k} & \text { otherwise }
\end{array}\right.
$$

8: $\quad k \leftarrow k+1$

9: $\quad$ Until the stopping criterion is reached $(k=N)$

\subsection{Genetic Algorithm}

Genetic algorithms are heuristic search and optimization techniques of solving problems inspired from genetic and natural evolution. These methods mimic the process of natural evolution and implement the optimization strategies by simulating evolution of population through natural selection.

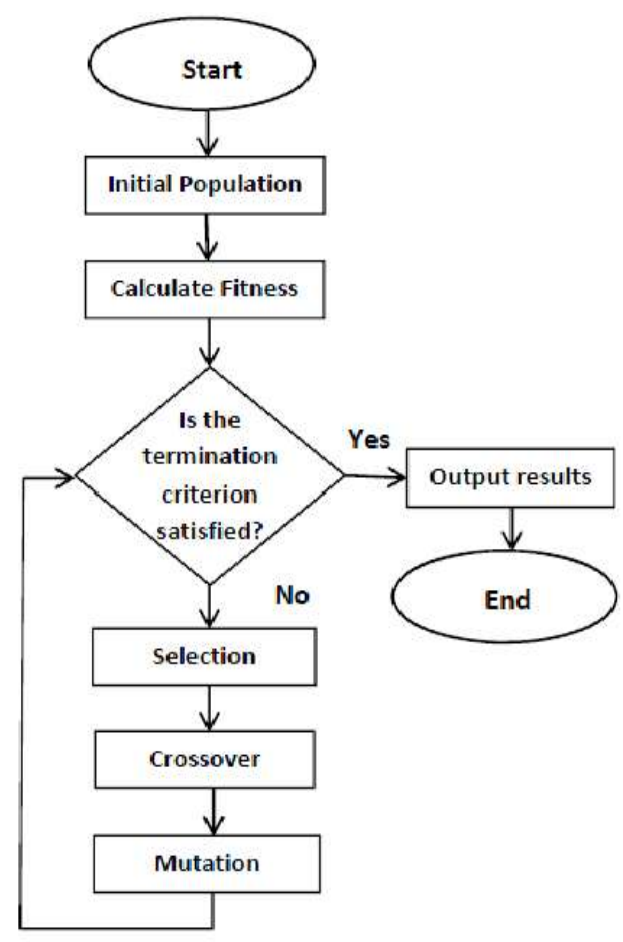

Figure 2. Genetic algorithm Flowchart

The basic steps of Genetic Algorithms are given by the flowcharts of figure 2.

\subsubsection{Selection}


A proportion of the existing population is selected during each successive generation in order to breed a new generation. The selection operator is to emphasize the good solutions and eliminate the bad solutions in a population. Individual solutions are selected through a fitness-based process, where fitter solutions as measured by fitness function.

\subsubsection{Crossover}

The crossover operator is used to combines the genetic material from parents order to produce children, during breeding. Since, only the good solutions are picked for breeding, during the selection procedure, the crossover operator mixes the genetic material, in order to produce children with even greater fitness and get the optimal solution. Figure 3 illustrate the principle of the crossover operation.

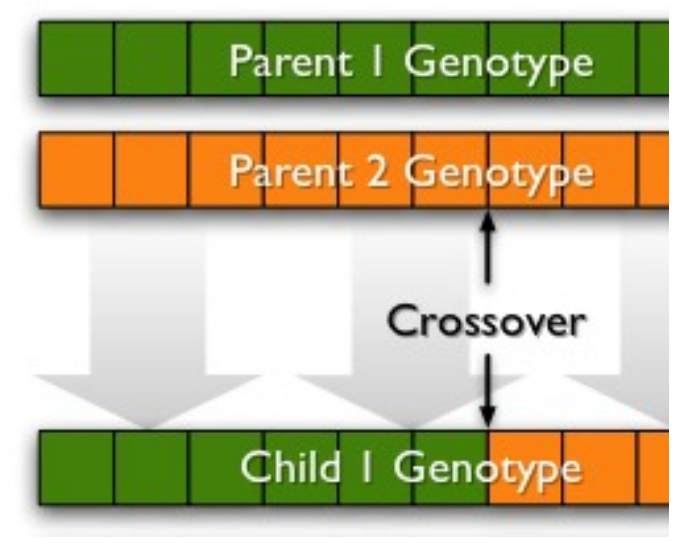

Figure 3. Crossover operation

Mutation operator maintains diversity in the population. Mutation is the inversion of a bit in a chromosome (Figure 4). This amounts to randomly change the value of a parameter of the individual. Mutations act as noise and impede the evolution of freeze. They ensure a local and global research, depending on the weight and the number of mutated bits. Moreover, they mathematically guarantee that the global optimum can be achieved. Figuratively speaking, the operation of the mutation can be explained by figure 4 .

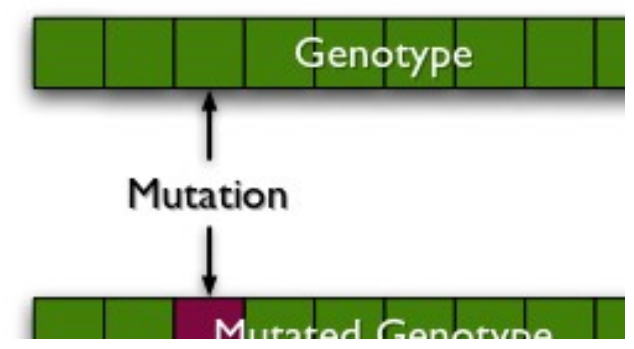

Figure 4. Mutation operation

\subsection{Objective function}


The basic step in applying optimization method is to choose the optimization criteria that are used to evaluate fitness. So that, we have chosen the following objective function $f$ in order to get the optimal value of the control:

$$
f=\operatorname{Max}_{i}(u(t))
$$

However, when the objective function (called also as fitness function) $f$ is minimized, a best maximum value of the control is obtained. This is explained by the fact of choosing a control law whose maximum value is the smallest as possible for all the possible iterations while guaranteeing the respect of actuator constraint previously fixed.

\section{Stabilization of switched linear systems}

\subsection{State feedback stabilization}

We consider in this section the following switched linear system:

$$
\dot{x}(t)=A_{\sigma} x(t)+B_{\sigma} \mathrm{u}(t), \quad \sigma \in\{1,2, \ldots, N\}
$$

We are interested in determining a state feedback control:

$$
u(t)=-K_{\sigma} x(t)
$$

The closed loop system is given by:

$$
\dot{x}(t)=\left(A_{\sigma}-B_{\sigma} K_{\sigma}\right) x(t)
$$

The search for the optimal state feedback gain $K_{\sigma}$ using differential evolution and genetic algorithm for the closed-loop switched linear system allows us to obtain the control low $u(t)$ that must satisfy two constraints:

\subsubsection{Constraint on the eigenvalues}

The oscillatory behavior in system response during the transitional regime is an important problem. In fact, the eigenvalues location affects the system response. In order to minimize oscillations, the eigenvalues of the closed-loop system must not exceed a limit negative real value $\beta$.

The constraint addressed in this paragraph can be stated as follows:

$$
\left(A_{\sigma}-B_{\sigma} K_{\sigma}\right)<\beta I
$$

where $I$ is an identity matrix with appropriate dimensions.

Remark. If it exists a common controller gain $K$ that satisfies the inequality (4.4) for all subsystems, then the stabilization by state feedback becomes global. 


\subsubsection{Constraint on the command}

In all systems, particularly switched systems, we have the problem of actuator limitations. In fact, actuators can only deliver a maximum value. In a practical point of view, this is manifested by limitations of actuators as the voltage generators, pressures, flow rates or any other kind of control... Otherwise, physically, the control will never be feasible and systems will not be controllable. As a consequence, commands have maximum value which must not be exceeded. So, the constraint on the control can be modeled as follows:

$$
\operatorname{Max}_{i}(u(t))<\gamma
$$

where $u(t)$ is the control, $i$ is the number of iteration and $\gamma$ is the limit value of the command.

\subsection{Output feedback stabilization}

Now, if the states are not available and only outputs $y(t)=C x(t)$ are measurable, it is possible to use the differential evolution and genetic algorithm in the case of stabilization by static output feedback controller. The problem addressed in this paragraph can be stated as follows:

Considering the static corrector given by the form of output feedback

$$
u(t)=-L_{\sigma} \mathrm{y}(t)
$$

The closed loop system is given by:

$$
\dot{x}(t)=\left(A_{\sigma}-B_{\sigma} L_{\sigma} C\right) x(t)
$$

The same as the state feedback, the search for the control low $u(t)$ using output feedback controller must also satisfy two constraints:

\subsubsection{Constraint on the eigenvalues}

The constraint can be stated as follows:

$$
\left(A_{\sigma}-B_{\sigma} L_{\sigma} C\right)<\beta I
$$

Remark. If it exists a common static observer gain $L$ that satisfies the inequality (4.7) for all subsystems, then the stabilization by output feedback becomes global.

\subsubsection{Constraint on the command}

As the state feedback controller, the constraint on the control is stated as follows:

$$
\underset{i}{\operatorname{Max}}(u(t))<\gamma
$$

\section{Algorithms implementation}


The proposed approach for stabilization of switched linear system by state feedback under control constraints can be described by the general algorithm which is summarized in Table 2.

Table 2. Stabilization algorithm by state feedback

\begin{tabular}{lc}
\hline 1: & Initialize the population randomly \\
2: & While (stop condition is not verified) do \\
3: & Generate the controller gain $K$ using DE or GA \\
4: & For N iterations do \\
4: & If $\left(A_{\sigma}-B_{\sigma} K\right)<\beta I$ do \\
5: & Minimize $f$ such as $f<\gamma$ \\
6: & End if \\
7: & End For \\
8: & Until N=Nmax
\end{tabular}

As the state feedback, we can directly deduce the output feedback stabilization algorithm by searching the static observer gain $L$ instead of searching for the gain $K$. Indeed, the algorithm obtained in the case of an output feedback is presented in table 3.

Table 3. Stabilization algorithm by output feedback

\begin{tabular}{lc}
\hline 1: & Initialize the population randomly \\
2: & While (stop condition is not verified) do \\
3: & Generate the controller gain $L$ using DE or GA \\
4: & For $N$ iterations do \\
4: & If $\left(A_{\sigma}-B_{\sigma} L C\right)<\beta I$ do \\
5: & End if \\
6: & End For \\
7: & $N=N_{\max }$ \\
8: & Until
\end{tabular}

\section{Simulation results and discussions}

In this section, we present the numerical results to prove the performance of the proposed methods. The first example illustrates the state feedback stabilization using differential evolution and genetic algorithm. The second one is devoted to the output feedback stabilization using evolutionary algorithms. All computations are implemented with MATLAB.

Example 1: State feedback stabilization

The performance of the developed stabilizing state feedback controllers using differential evolution and genetic algorithm was tested with the following switched linear system:

$$
\dot{x}(t)=A_{i} x(t)+B_{i} \mathrm{u}(t), \quad i \in\{1,2\}
$$


with

$$
\begin{array}{cc}
A_{1}=\left[\begin{array}{cc}
-0.73 & 1.23 \\
-0.40 & -0.66
\end{array}\right] \quad A_{2}=\left[\begin{array}{cc}
-0.11 & -0.74 \\
-0.50 & -1.22
\end{array}\right] \\
B_{1}=B_{2}=\left[\begin{array}{l}
1 \\
0
\end{array}\right] \quad \text { and } \quad x_{0}=\left[\begin{array}{c}
1 \\
-1
\end{array}\right]
\end{array}
$$

The chosen switched linear system is initially unstable. Indeed, the eigenvalues of $A_{1}$ and $A_{2}$ do not have all a negative real part. So that, a state feedback control law that stabilizes the switched linear system is designed.

Parameters values of the DE algorithm are setting as follows:

- number of iterations $N=1500$,

- $\quad$ scale factor $F=0.8$,

- $\quad$ crossover probability $C R=0.2$.

Parameters values of the GA algorithm are:

- number of iterations $N=1500$,

- mutation probability $m=0.05$,

- $\quad$ crossover probability $C R=1$.

The control constraints are fixed as follows:

- The real part of eigenvalues of $A_{i}-B_{i} K$ must be strictly less than -0.5 ,

- The maximum value of the control $u(\mathrm{t})$ must not exceed 1 .

By applying both differential evolution and genetic algorithm under conditions stated above, we get these following simulation results. Figure 5 shows the fitness functions of the given system. 


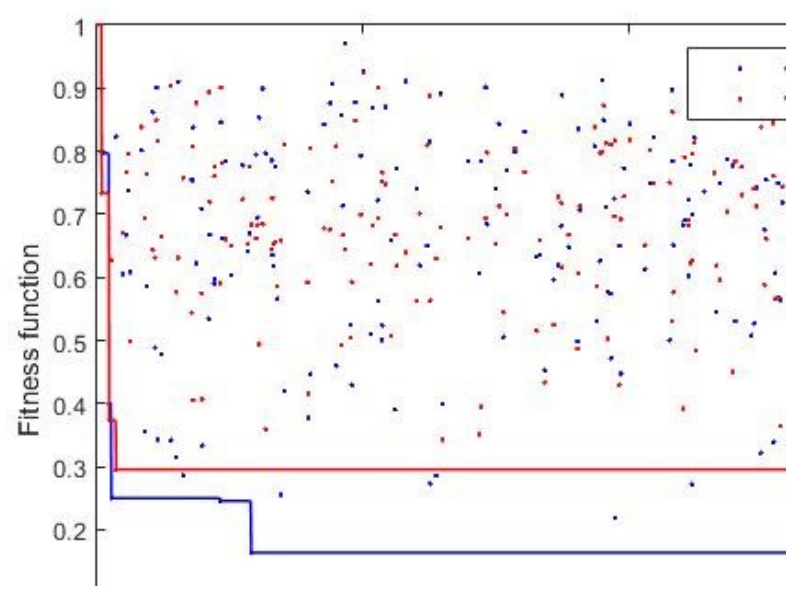

Figure 5. Fitness function

The evolution of the objective functions reported in Figure5 show that the objective functions value decreases substantially. Figure 6 plots the evolution of control behavior.

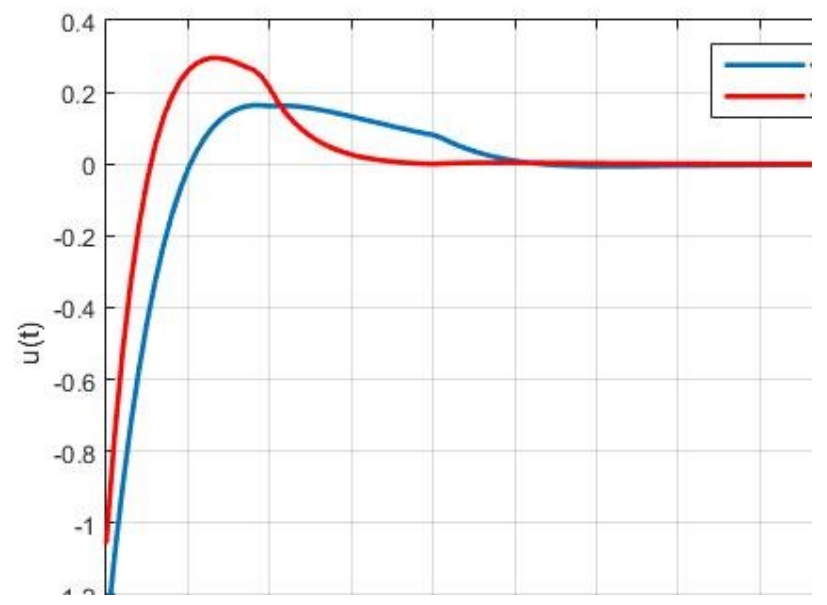

Figure 6. Control trajectories

Figure 7 draws the evolution of the switching signal.

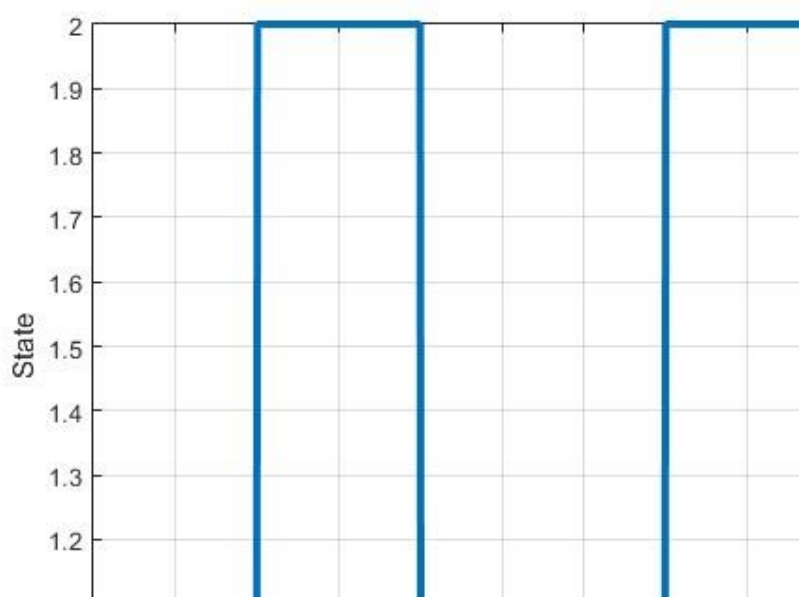

Figure 7. Switching signal 
The two components of the state are illustrated in Figure 8 and Figure 9.

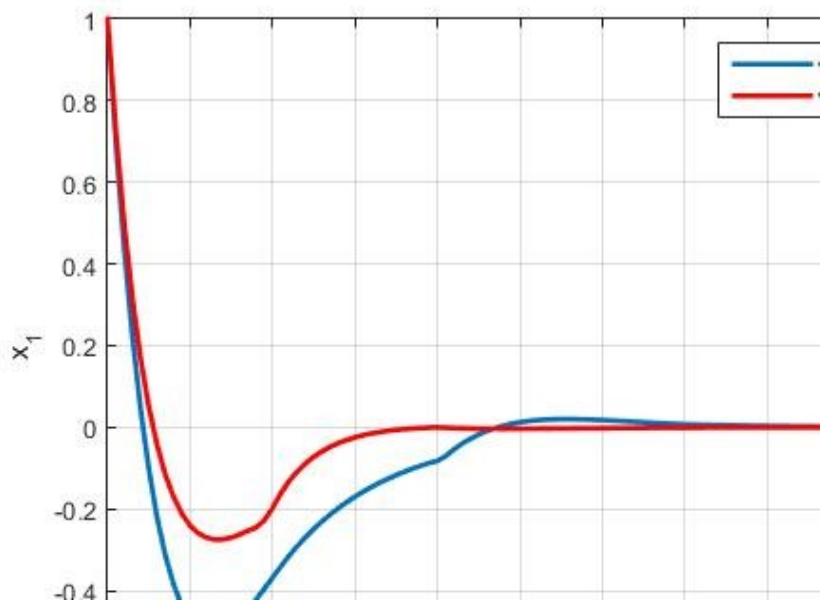

Figure 8. Evolution of the state $x_{1}$

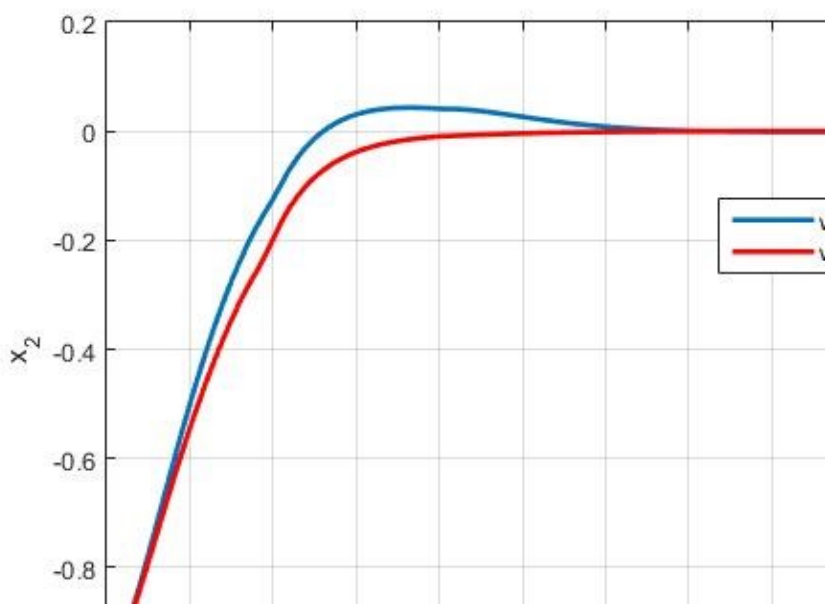

Figure 9. Evolution of the state $x_{2}$

Figure 10 shows the evolution of the norm of the state. 


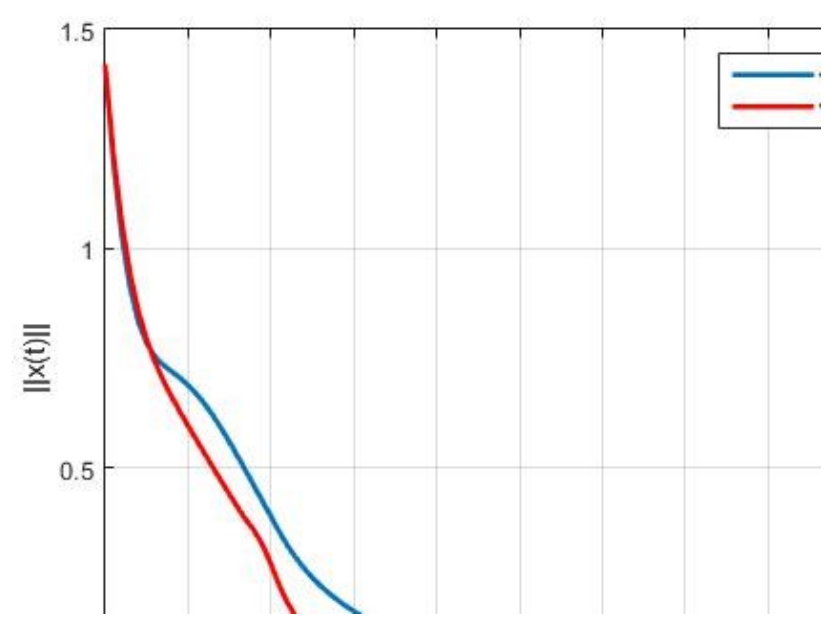

Figure 10. Evolution of the norm of the state

State feedback controllers are synthesized by using DE algorithm and GA algorithm. So that, the maximum value of control law $u_{\max }(t)$ and the static gain $K$ were computed in both cases and given in Table 4.

Table 4. State feedback stabilization results

\begin{tabular}{ccc}
\hline & Using DE & Using GA \\
\hline$u_{\max }(t)$ & 0.1635 & 0.2955 \\
\hline $\begin{array}{c}\text { The } \\
\text { corresponding } \\
\text { gain } K\end{array}$ & {$\left[\begin{array}{lll}0.6601 & -0.6532\end{array}\right]\left[\begin{array}{lll}1.0601 & 0.0098\end{array}\right]$} \\
\hline
\end{tabular}

The results show that in either algorithm, state feedback controllers can complete the desired purpose of stabilization of switched linear systems. Comparing the results obtained using differential evolution and those obtained using genetic algorithm reveals the existence of overshoot. In fact, system response with state feedback controller using DE algorithm has larger overshoot, while system response with state feedback controller using GA algorithm has the smallest overshoot. Both state feedback controllers produced good results quickly, but DE algorithm requires a long time to transition towards the optimal solution. Thus, system response using GA Algorithm is faster than system response using DE algorithm. However, with DE algorithm we obtain the optimal solution with the smallest value of control $u_{\max }(t)$ (Table 4). Indeed, having such smooth and flexible control facilitate its practical implementation that is why state feedback controllers based on DE algorithm proves its performance and effectiveness in solving the problem of stability of switched linear systems under control constraints.

Example 2: Output feedback stabilization 
The purpose of this example is to synthesize output feedback controllers to globally stabilize the switched linear system. The indicated controllers take in account the constraints imposed.

Considering the switched linear system described as follow:

$$
\left\{\begin{array}{l}
\dot{x}(t)=A_{i} x(t)+B_{i} u(t) \\
y(t)=C x(t)
\end{array} \quad i \in\{1,2\}\right.
$$

with

and

$$
\begin{gathered}
A_{1}=\left[\begin{array}{ll}
0.92 & 0.12 \\
0.04 & 0.84
\end{array}\right] ; \quad A_{2}=\left[\begin{array}{cc}
1.05 & 0.80 \\
-1.52 & 1.96
\end{array}\right] ; \\
B_{1}=B_{2}=\left[\begin{array}{l}
1 \\
0
\end{array}\right] ; \quad C=\left[\begin{array}{l}
0 \\
1
\end{array}\right] ; \\
x_{0}=\left[\begin{array}{c}
0.5 \\
-0.5
\end{array}\right] .
\end{gathered}
$$

Control constraints are stated as follows:

- The real part of eigenvalues of $A_{i}-B_{i} L C$ must be strictly less than -0.2 ,

- The maximum value of the control $u(\mathrm{t})$ must not exceed 2 .

Parameters values of the DE algorithm are setting as follows:

- $\quad N=2000$,

- $F=0.7$

- $\quad C R=0.1$.

Parameters values of the GA algorithm are:

- number of iterations $N=2000$,

- mutation probability $m=0.02$,

- $\quad$ crossover probability $C R=1$.

The following figures illustrate simulation results of our methodologies in order to stabilize the switched linear system by output feedback with control constraints. We can see in Figure 11 the evolution of the fitness function. 


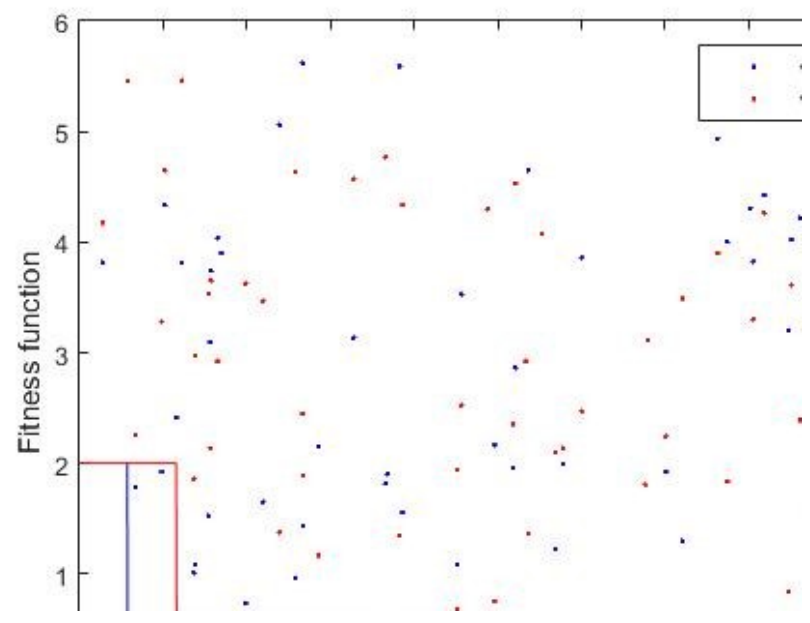

Figure 11. Fitness function

Figure 12 draws the evolution of the control behavior.

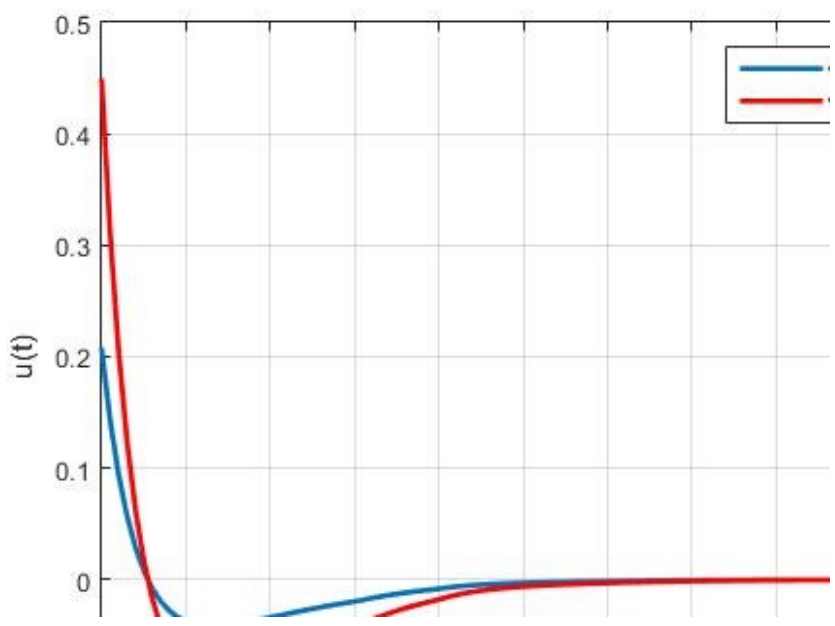

Figure 12. Control trajectories

Figure 12 shows that the output feedback controllers success to stabilize the switched linear system. Figure 13 plots the evolution of switching signal. 


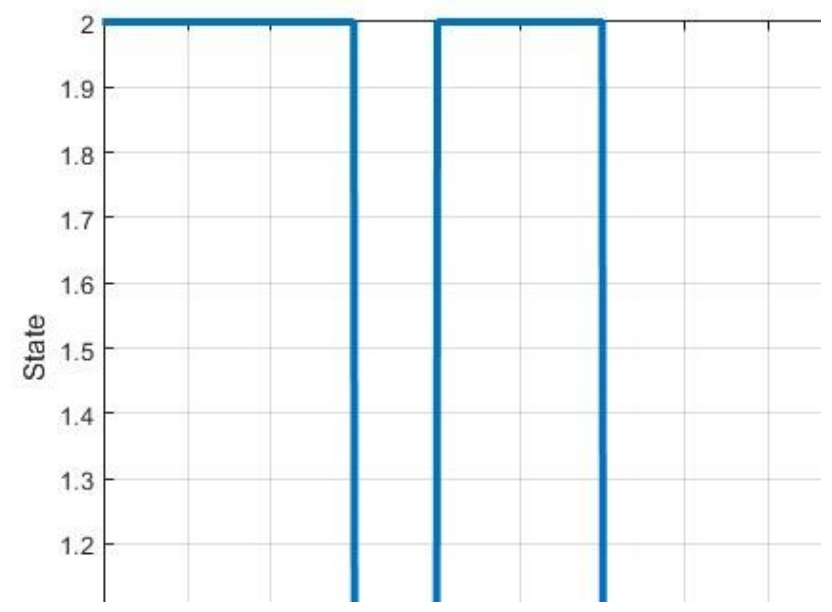

Figure 13. The switching signal

The two components of the state are illustrated in Figure 14 and Figure 15. Figure 16 shows the evolution of the norm of the state.

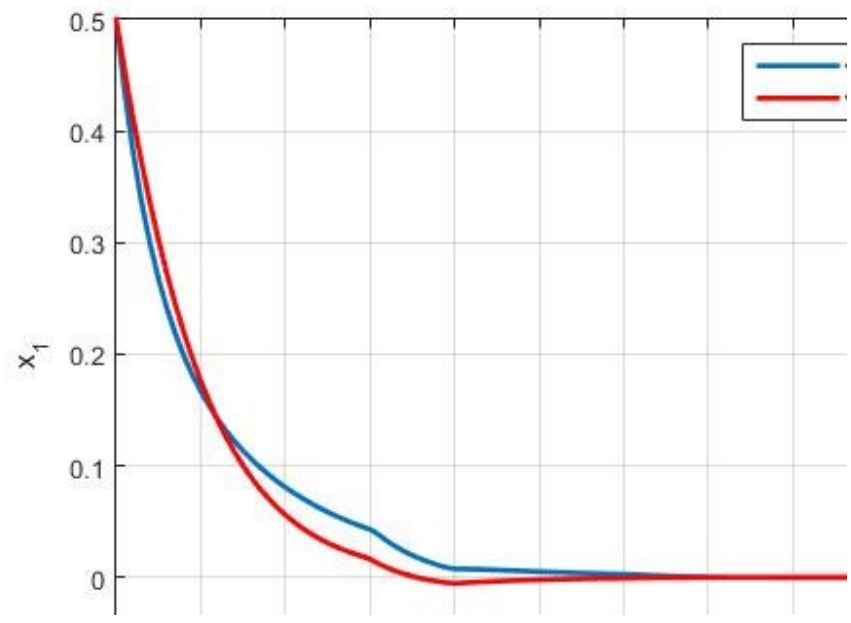

Figure 14. Evolution of the stats $x_{1}$ 


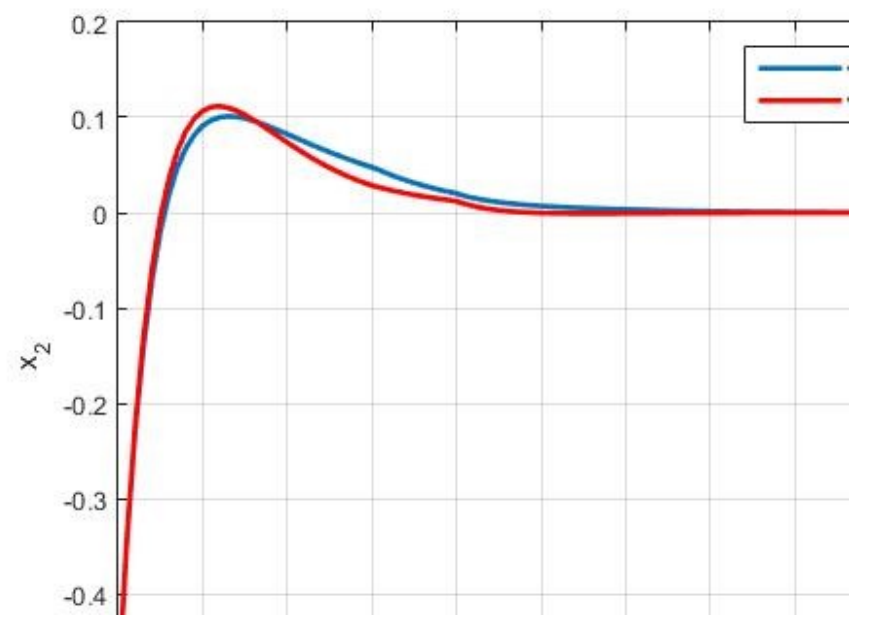

Figure 15. Evolution of the stats $x_{2}$

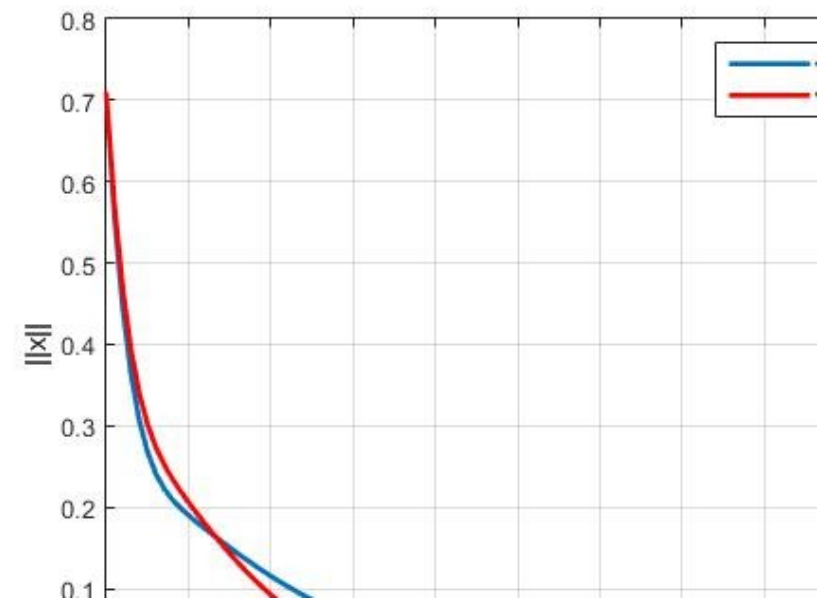

Figure 16. Evolution of the norm of the state

After applying the two algorithms, we obtain the maximum value of the control law $u_{\text {max }}(t)$ and the corresponding static gain $L$ presented in the table 5 .

Table 5. Output feedback stabilization result

\begin{tabular}{ccc}
\hline & Using DE & Using GA \\
\hline$u_{\max }(t)$ & 0.2058 & 0.4472 \\
\hline $\begin{array}{c}\text { The } \\
\text { corresponding } \\
\text { static gain } L\end{array}$ & 0.4117 & 0.8945 \\
\hline
\end{tabular}

The simulation results presented above proved that our approaches are efficient tools for the resolution of the stabilization problem for the switched linear systems by searching for the optimal 
static gain $L$ using evolutionary algorithms. A major advantage in these approaches is the explicit design of the control law. System response using output feedback controllers based on differential evolution and genetic algorithm give good performances and it can be successfully used to stabilize switched linear systems. Comparing the results obtained using differential evolution and those obtained using genetic algorithm, we can deduce that output feedback controller using DE gives the optimal solution with the lowest value of control $u_{\max }(t)$ and has the smallest overshoot. However, output feedback controller using GA has the faster response.

\section{Conclusions}

The problem of state feedback stabilization and output feedback stabilization of switched linear systems under control constraints is solved by searching stabilizing control law based on the differential evolution and genetic algorithm. Our methodologies are used to optimize the performances of this class of system and to solve multi objective problems. To demonstrate the performance of our proposed approaches, stabilizing state feedback and output feedback controllers based on evolutionary algorithms are implemented and compared.

Conflicts of Interest: The authors declare no conflict of interest.

\section{References}

1. Agrachev AA, Liberzon D. (2001) Lie-algebraic stability criteria for switched systems. SIAM J Control Opt; 41:253-69.

2. Cheng D, Guo L, Huan J. (2003) On quadratic Lyapunov functions. IEEE Transaction Automatic Control; 48:885-90.

3. Dayawansa WP, Martin CF. (1999) A converse Lyapunov theorem for a class of dynamical systems which undergo switching. IEEE Transaction Automatic Control; 44:751-60.

4. Daafouz J, Riedinger P, Iung C. (1999) Stability analysis and control synthesis for switched systems: a switched Lyapunov function approach. IEEE Transaction Automatic Control; 47:1883-7.

5. D. Liberzon, A . Morse. (1999) Basic problems in stability and design of switched systems. IEEE Control Systems Magazine, 19(5): 59-70.

6. M. Branicky. (1998) Multiple Lyapunov functions and other analysis tools for switched and hybrid systems. IEEE Transaction on Automatic Control, 43(4): 475-482.

7. J. Hespanha, A. Morse. (1999) Stability of switched systems with average dwell time. Proceedings of of the 38th Conference on Decision and Control: 2655-2660.

8. G. Zhai,B. Hu, K. Yasuda, et al. (2000) Stability analysis of switched systems with stable and unstable subsystems: An average dwell time approach. Proceedings of the American Control Conference: 200-204.

9. D. Liberzon. (2003) Switching in systems and control. Boston: Brikhauser.

10. Lin, H., and Antsaklis, P.J., (2009) Stability and Stabilisability of Switched Linear Systems: A Survey of Recent Results', IEEE Transactions on Automatic Control, 54, 308-322.

11. Rodrigo H. Ordonez-Hurtado and Manuel A. Duarte-Mermoud, (2011) A Methodology for Determining the Non-Existence of Common Quadratic Lyapunov Functions for Pairs of Stable Systems, Fifth International Conference on Genetic and Evolutionary Computing.

12. C.Scherer, P.Gahinet, and M.Chilali. (1997) Multi objective output-feedback control via LMI optimization, IEEE Transactions on Automatic Control, VOL. 42, NO. 7.

13. B. Lincoln and A. Rantzer. (2006) Relaxing dynamic programming. IEEE Transactions on Automatic Control, 51(8):1249-1260.

14. C. Seatzu, D. Corona, A. Giua, and A. Bemporad. (2006) Optimal control of continuous-time switched affine systems. IEEE Transactions on Automatic Control, 51(5):726-741. 
15. H. Axelsson, Y. Wardi, M. Egerstedt, and E.I. Verriest. (2015) Gradient descent approach to optimal mode scheduling in hybrid dynamical systems. Journal of Optimization Theory and Applications, 136(2):167-186, 2008. Preprint submitted to Automatica November 23.

16. S.C. Bengea and R. A. DeCarlo. (2005) Optimal control of switching systems. Automatica, 41(1):11-27.

17. P. Colaneri, J.C. Geromel, and A. Astolfi. (2008) Stabilization of continuous-time switched nonlinear systems. Systems \& Control Letters, 57(1):95-103.

18. G.S. Deaecto, J.C. Geromel, and J. Daafouz. (2011) Switched state-feedback control for continuous time-varying polytopic systems. International Journal of Control, 84(9):1500-1508.

19. P. Riedinger and I.-C. Morarescu. (2014) A numerical framework for optimal control of switched input affine nonlinear systems subject to path constraint. Mathematics and Computers in Simulation, 95(0):63 77.

20. Juing-Shian Chiou and Chun-Ming Cheng, (2009) Stabilization analysis of the switched discrete-time systems using Lyapunov stability theorem and genetic algorithm.

21. R.H. Ordóñez-Hurtado and M.A. Duarte-Mermoud, (2012), Finding common quadratic Lyapunov functions for switched linear systems using particle swarm optimization, International Journal of Control.

22. M.A. Duarte-Mermoud, R.H. Ordóñez-Hurtado and P. Zagalak, (2012), A method for determining the non-existence of a common quadratic Lyapunov function for switched linear systems based on particle swarm optimization, International Journal of Systems Science.

23. Liberzon, D., and Tempo, R., (2004) Common Lyapunov Functions and Gradient Algorithms, IEEE Transactions on Automatic Control, 49, 990-994.

24. Houssem HOSNI, Walid BEN MABROUK, and Noureddine LIOUANE. "Stabilization of switched linear systems using differential evolution algorithm." International Journal of Applied Engineering Research 10.18 (2015): 39285-39289.

25. K. Price, R. Storn, and J. Lampinen, (2005) Differential Evolution: A Practical Approach to Global Optimization, ser. Natural Computing Series. Springer-Verlag.

26. Zaharie, D. (2003) Control of population diversity and adaptation in differential evolution algorithms. In: Matousek, R., Osmera, P. (eds.) 9th International Conference on Soft Computing, MENDEL.

27. Swagatam Das, Ponnuthurai Nagaratnam Suganthan, (2011) Differential Evolution: A Survey of the State-of-the-Art. IEEE Transactions on evolutionary computation, vol. 15, No. 1. 